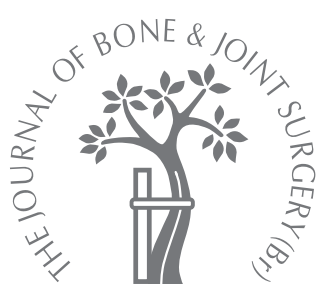

J. K. Dickson,

L. C. Biant

From The Peripheral

Nerve Injuries Unit,

The Royal National

Orthopaedic

Hospital, Stanmore, England

\title{
A good outcome following complete injury of the brachial plexus
}

\author{
LONG-TERM ANALYSIS OF THE MANAGEMENT OF TWO \\ PATIENTS
}

\begin{abstract}
Restoration of hand function is rarely achieved after a complete closed traction lesion of the supraclavicular brachial plexus. We describe the injury, treatment, rehabilitation and longterm results of two patients who regained good function of the upper limb and useful function in the hand after such an injury. Successful repairs were performed within six days of injury. Tinel's sign proved accurate in predicting the ruptures and the distribution of pain was accurate in predicting avulsion. The severe pain that began on the day of injury resolved with the onset of muscle function.

Recovery of muscle function preceded recovery of sensation. Recovery of the function of $C$ and $A \delta$ fibres was the slowest of all.
\end{abstract}

J. K. Dickson, MBBChir MA (Cantab), MRCS, Senior House Officer

The Peripheral Nerve Injuries Unit

The Royal National

Orthopaedic Hospital, Brockley

Hill, Stanmore, Middlesex HA7

4LP, UK.

- L. C. Biant, FRCSEd(Tr \& Orth), MS, Consultant Trauma and Orthopaedic Surgeon The Royal Infirmary of Edinburgh, Little France Crescent, Old Dalkeith Road Edinburgh EH16 4SA, UK.

Correspondence should be sent to $\mathrm{Mr}$ J. K. Dickson; e-mail: john.k.dickson@gmail.com

(C)2010 British Editorial Society of Bone and Joint Surgery doi:10.1302/0301-620X.92B4 $23291 \$ 2.00$

$J$ Bone Joint Surg $[\mathrm{Br}]$ 2010;92-B:540-4.

Injury to the brachial plexus can lead to debilitating loss of function and severe pain. The pain can be difficult to manage and can be a barrier to rehabilitation. It occurs in the insensate arm and is described as 'crushing' or 'burning' in nature. The patient may experience superimposed 'lightning shock' sensations which correspond to the dermatomes of the avulsed nerve roots. ${ }^{1}$ The majority of patients affected by these injuries are men of working age, and $82 \%$ of such cases result from motorcycle accidents. ${ }^{2,3}$ Early surgical intervention correlates with a reduction in pain., ${ }^{4,5}$

The initial clinical assessment can distinguish between pre- and post-ganglionic lesions. Pre-ganglionic lesions have a poorer prognosis, as many represent complete avulsion of the nerve roots from the spinal cord. Complete brachial plexus lesions are associated with poor long-term function and chronic pain. ${ }^{6}$ Over 1370 injuries to the brachial plexus have been treated at our unit in the last 16 years. We analysed in detail the long-term outcome in two patients with complete lesions who had a result that was better than expected, in order to identify the factors that made the end result so favourable.

\section{Patient 1}

A 23-year-old right-handed female sustained multiple injuries to the left upper and lower limbs in a road traffic accident when she was a pillion passenger on a motorcycle. Closed fractures of the left humerus, radius and ulna were treated by open reduction and internal fixation. The radial nerve was explored and found to be stretched and contused, but not ruptured. Dislocation of the left knee was treated by staged repair of the ligaments. The diagnosis of a complete lesion of the left brachial plexus was made on admission, and she was transferred to a specialist peripheral nerve injury unit as soon as her other injuries allowed safe transport.

Five days after injury she was in intense pain. Her visual analogue score was 10/10, the peripheral nerve injury score ${ }^{7} 4 / 4$, and she had not responded to carbamazepine, amitriptyline or opiates. The pain was severe and had started immediately after the injury; she described it as a constant burning pain throughout the hand. There was superimposed 'lightning-like shocks' of shooting pain of exquisite severity from the elbow along the radial side of the forearm to the thumb, occurring approximately 12 times every hour. There were bruising and linear abrasion in the posterior triangle of the neck. Loss of sensation extended from the dermatomes of C5 to those of T1. The ipsilateral hemi-diaphragm and the serratus anterior were functioning. All other muscles innervated by C5 to T1 were paralysed. There was no BernardHorner syndrome. A strong, painful Tinel's sign was elicited by percussion in the posterior triangle of the neck, with radiation of intense parasthesiae to the radial aspect of the forearm (C5, C6), the dorsal and palmar aspect of the hand $(\mathrm{C} 7, \mathrm{C} 8)$, and to the medial aspect of the forearm (T1). CT myelography 


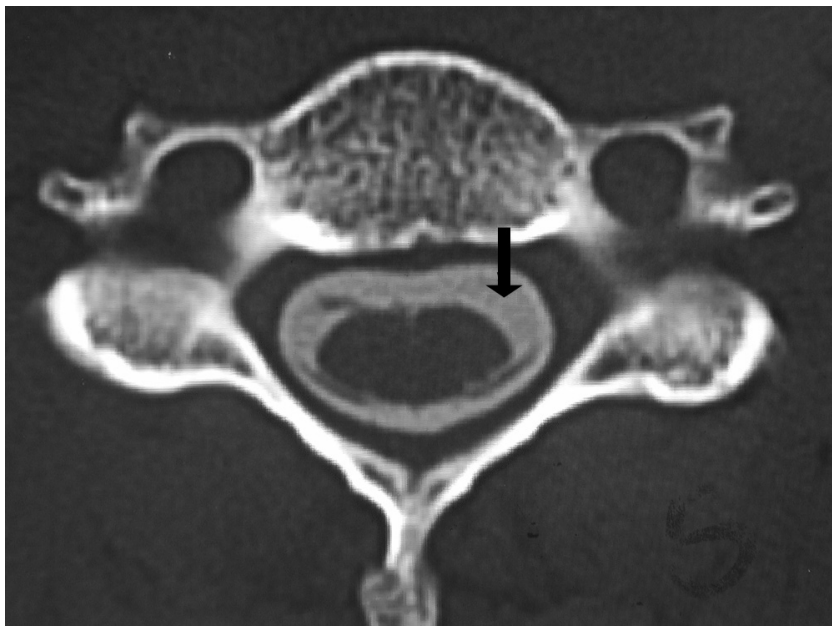

Fig. 1

High-resolution CT myelogram showing isolated avulsion of the left C6 ventral root (arrow) in patient 1.

showed intradural injury to the roots of $\mathrm{C} 6$, which confirmed the clinical diagnosis of ruptures of $\mathrm{C} 5, \mathrm{C} 7, \mathrm{C} 8$ and T1 and avulsion of C6 (Fig. 1).

Six days after injury the brachial plexus was explored, and all the proximal and distal stumps were identified. The condition of the ruptured spinal nerves of C5, C8 and T1 was good, but that of C7 was poor and C6 was avulsed. Somatosensory evoked potentials recorded from C5, C8 and $\mathrm{T} 1$ were normal in amplitude and form. The trace for C7 was abnormal in form and diminished in amplitude. The gap between the prepared proximal and distal faces was $7 \mathrm{~cm}$ at the widest point. A map was drawn of the proximal and distal faces, marking out the arrangement of the nerve bundles and indicating their size.

Cutaneous nerves from the injured ipsilateral limb and both sural nerves were harvested, with a total length of $120 \mathrm{~cm}$. The repair was based mainly on the healthiest stumps (C5, C8 and T1). The spinal accessory nerve was transferred to the distal suprascapular nerve. There were eight grafts placed from C5, three to C5 itself and five to C6. The ventral root of $\mathrm{C} 6$ was repaired by placing a graft between it and the most anterior bundle of proximal C5. The dorsal component of $\mathrm{C} 6$ was repaired by four grafts based on C5 after excision of the dorsal root ganglion. Repair of C7 was achieved with four grafts. The lower trunk was repaired with three grafts from $\mathrm{T} 1$ and five from $\mathrm{C} 8$. The grafts were held in place using fibrin sealant (Tisseel; Baxter, Newbury, United Kingdom).

After operation, the arm was immobilised with the shoulder in adduction and medial rotation, using a sling with a strap around the waist and a soft collar. ${ }^{7}$ After five weeks she was readmitted to hospital for five days for mobilisation and rehabilitation. Further post-operative care was guided by the following principles: continued mobilisation, the treatment of pain, return to work and

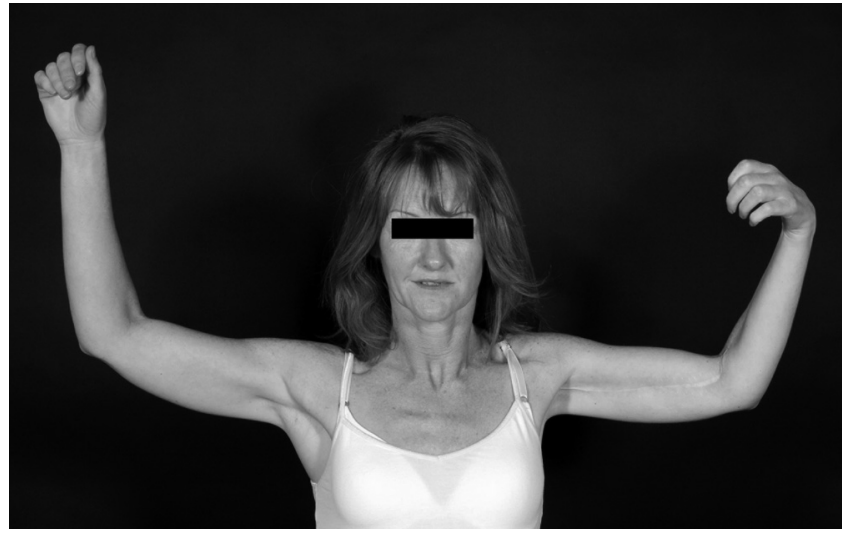

Fig. 2a

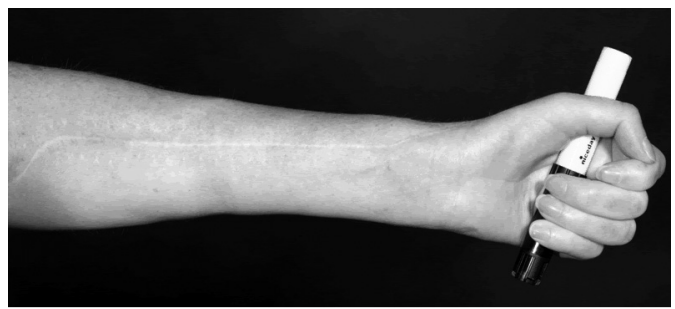

Fig. $2 b$

Photograph of patient 1 showing a) function at eight years after injury and $b$ ) hand function four months after transfer of flexor carpi ulnaris to extensor carpi radialis brevis, performed eight years after injury.

driving, and a positive and encouraging approach to the patient.

The pain remained severe for 15 months and began to improve concurrent with recovery of muscle function. The shooting pain had disappeared by 18 months from injury and by eight years she was free of pain.

At eight months there was recovery of function into the supraspinatus and the clavicular head of pectoralis major. A strong Tinel's sign was elicited $32 \mathrm{~cm}$ distal to the level of the rupture, indicating the level of regeneration. At 13 months, recovery was detected in the biceps brachii and flexor digitorum superficialis. Tinel's signs were elicited over the median, radial and ulnar nerves at $40 \mathrm{~cm}$ distal to the level of the rupture. By 36 months, activity was apparent in the thenar muscles. Eight years after injury (Fig. 2a) she had recovered a full range of shoulder movement, powerful flexion and extension at the elbow, and flexion of the wrist and digits (Fig. 2b).

Quantitative sensory testing was performed at 15 months using a range of non-invasive quantitative measures that have been previously validated in adults. ${ }^{8,9}$ There was no recovery in the hand, nor any recovery of cholinergic (sympathetic) sweating at this stage. At 46 months the thresholds to von Frey hairs had improved, and thermal thresholds were demonstrable, albeit elevated (Table I). Recovery was noted to be slower for somatic afferent fibres 
Table I. Patient 1 at a follow-up of 46 months showing the results of quantitative sensory testing in the left arm using a range of non-invasive measures ${ }^{8,9}$

\begin{tabular}{llll}
\hline & Warm threshold $\left({ }^{\circ} \mathbf{C}\right)$ & Cool threshold $\left({ }^{\circ} \mathrm{C}\right)$ & Monofilament thresholds \\
\cline { 2 - 4 } Normal values & $(<\mathbf{3 . 9})$ & $(<2.6)$ & $(<3)$ \\
\hline C5 & 4.4 & 2.5 & 4 \\
C6 & 14.7 & 3.6 & 6 \\
C7 & 8.0 & 5.5 & 4 \\
C8 & - & - & 8 \\
\hline
\end{tabular}

than for the larger efferent motor fibres. At eight years the von Frey thresholds had improved still further and were recorded as grade 3 in C5, grade 5 in C6, grade 4 in C7 and grade 3 in T1. By this time, she had accurate localisation to the digit and the return of sweating. She returned to work after 15 months when she had started driving a modified car. Her recovery was hampered by symptoms of entrapment neuropathy in the right upper limb which may have been provoked by compensatory overuse. These were resolved by decompression of the median nerve.

\section{Patient 2}

A 28-year-old right-handed male was injured when he cycled into the path of an oncoming car. He sustained a complete injury to the right brachial plexus with fractures of the ipsilateral radius, ulna and hand. The arm was flail. The trapezius, rhomboids and serratus anterior were functioning, but there was no muscle activity below this level. Sensation was absent from below the shoulder laterally and above the elbow in the anterior plane. Sensation in the axilla was present but altered. There was a strong positive Tinel's sign for C6 in the posterior triangle. There was no Bernard-Horner syndrome. Pain had commenced instantly on injury; it was constant, burning and crushing in nature, felt in the forearm and hand. It was severe and scored by the patient as $10 / 10$ on a visual analogue scale. There was no shooting pain. He underwent immediate open reduction and internal fixation of the fractures of the radius, ulnar and of the metacarpals.

The brachial plexus was explored six days after the injury. Nerves C5 to T1 were all ruptured, and C5 was ruptured at the level of the ganglion. The other injuries were postganglionic. Intra-operative somatosensory evoked potentials from electrodes over the neck showed reproducible and consistent traces at all five levels, with the strongest at C6 and $\mathrm{C} 7$, weaker at C8 and T1, and weakest at C5, in keeping with the physical findings. The phrenic nerve was conducting normally.

All five spinal nerve ruptures were grafted using $120 \mathrm{~cm}$ of nerve graft raised by harvesting the sural nerves, the ipsilateral superficial radial nerve and the medial cutaneous nerve of the forearm using 20 segments of $5 \mathrm{~cm}$ to $6 \mathrm{~cm}$ in length. The accessory nerve was transferred to the suprascapular nerve. Four grafts were used to repair C5, six for $\mathrm{C} 6$ and five for $\mathrm{C} 7$. A further five grafts were placed from
C8 and T1 to the lower trunk. The condition of all the proximal stumps was good, and an accurate topographical match was possible. Post-operatively the pain began to ease with improvement of function and by 24 months he was free of pain.

At six months the Tinel's sign had advanced $18 \mathrm{~cm}$ distally and function had started to return in supraspinatus and pectoralis major. By eight months the first flickers of recovery were detected in the forearm flexors. At 24 months he had a full range of movement in the shoulder, with no contractures. At this time, flexion of the elbow was MRC grade 4 and of the fingers grade 3 . At 60 months there was no wrist extension and no function in the intrinsic muscles of the hand. At 15 years he had motor power of MRC grade 5 in the muscles of the rotator cuff, the deltoid, the biceps brachii, the triceps, flexor carpi ulnaris and the finger flexors (Fig. 3). The abductor digit minimi functioned at grade 2 , and the opponens pollicis at grade 3 (Fig. $3 \mathrm{~b}$ ). The quantitative sensory testing studies at this time are shown in Table II.

\section{Discussion}

With a complete injury to the brachial plexus, rupture and avulsion are followed by rapid cell death caused by interruption of the supply of neutrotrophic factors and associated vascular trauma. ${ }^{10}$ The supportive Schwann cell network stops being responsive to the regenerating axons after a period of only several months. ${ }^{11}$ In both of the cases studied, urgent exploration and repair was performed at six days, resulting in a good long-term outcome with resolution of pain and some recovery of useful function into the hand.

It is rare for patients to regain such function after a complete brachial plexus injury, and there are few cases reported in the literature. ${ }^{12}$ In cases of rupture of the upper nerves with avulsion of C7, C8 and T1, or of C8 and $\mathrm{T} 1$, the ipsilateral ulnar nerve has been used as a free vascularised graft to reconnect proximal stumps with the sources of the median nerve distally. However, in a series of 65 such cases, useful hand function was recovered in only three. ${ }^{12}$ In two of these patients, both aged 17 , surgery was undertaken four days after injury. In the third case, a child aged three years, the operation was performed eight weeks after injury. Transfer of the contralateral seventh cervical nerve, via an interposed free 
Table II. Patient 2 at 180 months after injury showing quantitative sensory testing in the right arm using a range of non-invasive measures ${ }^{8,9}$

\begin{tabular}{|c|c|c|c|c|c|}
\hline & Warm threshold $\left({ }^{\circ} \mathrm{C}\right)$ & Cool threshold $\left({ }^{\circ} \mathrm{C}\right)$ & Monofilament thresholds & Pinprick sensation & Vibration thresholds \\
\hline Normal values & $(<3.9)$ & $(<2.6)$ & $(<3)$ & & $(<10)$ \\
\hline $\mathrm{C} 5$ & 2.9 & 4.5 & 9 & Blunt & Shoulder - 14 \\
\hline $\mathrm{C} 6$ & 5.0 & 8.6 & 8 & Tingling & Elbow - 48 \\
\hline $\mathrm{C7}$ & 5.0 & 3.2 & 10 & Tingling & Wrist - 42 \\
\hline $\mathrm{C} 8$ & 7.4 & 2.8 & 14 & Tingling & Thumb - 32 \\
\hline T1 & 10.3 & 6.3 & 2 & Tingling & $\begin{array}{l}\text { Middle finger - } 35 \\
\text { Little finger - } 42\end{array}$ \\
\hline
\end{tabular}

N. B. Sweating was found to be 29 and 30 units in the right and left palms, respectively. At the fingers he was able to sense movement but not direction.

vascularised nerve graft, was introduced by $\mathrm{Gu}_{\mathrm{e}} \mathrm{al}^{13}$ as a method of re-innervating the median nerve, and one case in this series showed remarkable recovery into the hand. Other approaches to regaining hand function have been proposed, and Doi ${ }^{14}$ has developed a universal reconstructive procedure for total paralysis of the upper limb using a double free-muscle transfer technique.

In both of the cases described here the level and extent of injury were determined at the initial presentation by clinical examination alone. There was clear evidence of forcible separation of the forequarter from the trunk. Both patients demonstrated signs of the violence of the injury by bruising in the posterior triangle, and by linear abrasions extending from the chin to the shoulder. Both experienced immediate and severe pain, which was expressed throughout the insensate limb. The pain was constant and burning in quality. In the first patient there was also convulsive, lightninglike shooting pain, radiating to the radial aspect of the forearm towards the thumb. This finding aided accurate diagnosis of avulsion of the sixth cervical nerve. A strong Tinel's sign shows that the axons have been ruptured, and was demonstrated in both patients at the initial presentation. The significance of Tinel's sign in closed traction lesions was recognised by Landi et $\mathrm{al}^{15}$ in 1980 . The extent of the injury was apparent by loss of skin sensibility throughout the territory of C5 to T1. In both cases, only the phrenic nerve and the nerve to serratus anterior were conducting before operation.

In patient $1, \mathrm{CT}$ myelography confirmed the diagnosis of avulsion of the C6 root. CT scanning with contrast enhancement has been shown to have a $75 \%$ correlation with the surgical findings and significantly better diagnostic accuracy than conventional myelography. ${ }^{16}$ MRI has also been shown to be a useful investigation that can contribute to the planning of surgery. ${ }^{17}$

The operative repair in both cases involved an initial assessment of the stumps based on their appearance and the somatosensory evoked potentials. The stumps were then prepared by resection back to healthy faces. No more than $3 \mathrm{~mm}$ of tissue required resection. A sketch map was made of the proximal and distal faces to facilitate planning. The whole of the plexus was repaired. If the spinal nerve had been avulsed, or where its condition was poor, the distal

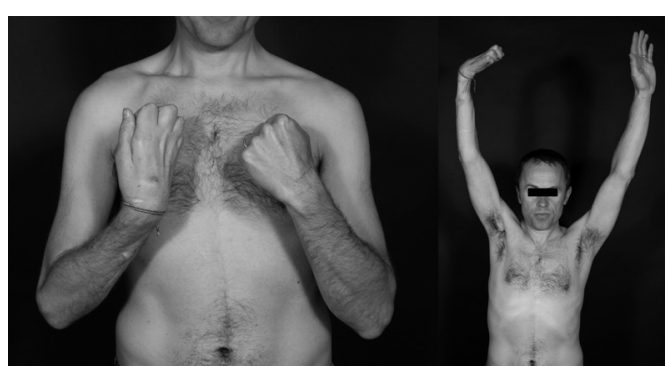

Fig. 3a

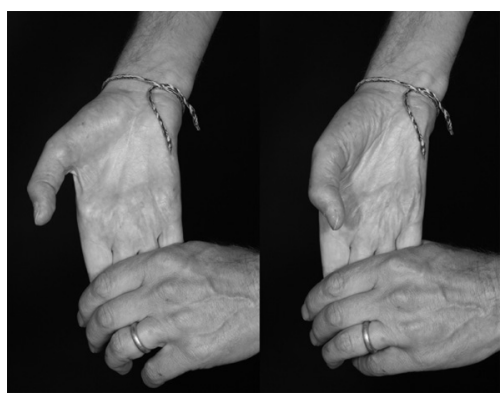

Fig. 3b

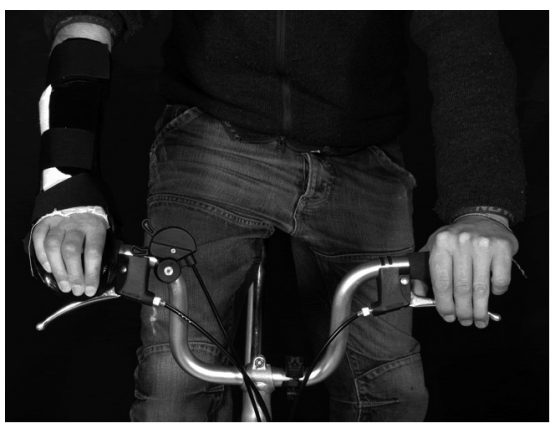

Fig. 3c

Photograph of patient 2 at 15 years after exploration and repair of a complete lesion of the brachial plexus showing a) muscle function at the shoulder, elbow and wrist; b) function in the intrinsic muscles of the hand, particularly opponens pollicis; and c) overall function of the upper limb.

stumps of these nerves were repaired by grafts from adjacent healthy ruptures. The aim throughout was to achieve an accurate topographical match. The gaps between the 
proximal and distal faces varied from $3 \mathrm{~cm}$ to $7 \mathrm{~cm}$, but were reduced by drawing the distal stumps back up into the posterior triangle. Transfer of the accessory to the suprascapular nerve was performed using suture and fibrin sealant. All other grafts were held in position using fibrin sealant alone.

Nerve regeneration is centrifugal, and the first signs of recovery were seen in the most proximal muscles. The relief of pain in these cases occurred with the onset of muscle function and was critical for rehabilitation. Reinnervation of the flexor muscles of the forearm was apparent at around eight months, and of the small muscles of the hand at 32 months. However, the strength and stamina of the re-innervated muscles continued to improve for over ten years. The pattern of recovery demonstrated improvement in the larger efferent fibres ahead of the somatic afferent fibres with the autonomic fibres last. Sensation to light touch recovered before warm and cool sensation. The thresholds to stimulus diminished over many months, approaching normal levels at about eight years after repair. The early hyperalgesia to warm and cool stimulus improved with recovery. Recovery of post-ganglionic sympathetic vaso- and endo-motor function was slower than somatic afferent and efferent recovery. The larger fibres seemed to recover more rapidly. This may reflect the different sizes of the cell bodies involved and the shorter distance for regeneration to the muscle than to the skin. It is interesting to note that this pattern of recovery is in striking contrast to the situation in obstetric brachial plexus lesions, where sensory recovery has been reported to exceed both motor and cholinergic sympathetic recovery. ${ }^{9}$

It is important to detect and manage other associated injuries appropriately. Such severe injuries will frequently be associated with a second-level lesion. This is illustrated by patient 1 , where the radial nerve was examined during the initial open reduction and internal fixation of the fracture of the humerus and found to be contused and stretched, but still in continuity. Such information can be helpful in planning the subsequent management and rehabilitation. Associated injuries must take priority, in particular, those to the head, chest and spine. Injuries affecting the limb can complicate the management and recovery of the plexus lesion. Patient 2 had sustained open fracture dislocations of the heads of the fourth and fifth metacarpals, as well as a fracture to the proximal phalanx of the ring finger and compound fractures of the radius and ulna. At five years the metacarpophalangeal joints were released and transfer of flexor carpi radialis to the extensor carpi radialis brevis was performed. This operation failed, and raises a question about the most appropriate timing for such reconstruction. It may be optimal to delay performing such a reconstruction until recovery has entirely plateaued. Patient 1 underwent a tendon transfer at eight years, with a much better result (Fig. 2b).

These cases both had a good long-term outcome after prompt diagnosis and urgent repair, and the patients were well motivated. The sooner the distal segment is connected to the cell body and the proximal segment, the better the outcome is likely to be. ${ }^{18}$ The relief of pain was the key to enabling recovery and re-integration, and this improved with re-innervation of the muscles. ${ }^{19}$

We thank Professor R. Birch, who performed the surgery and reviewed the paper. Professor P. Anand and Dr V. P. Misra performed the quantitative sensory testing. Mr G. Thomas (Derriford Hospital, Plymouth) initially treated Patient 1 and $\mathrm{Mr}$ J. Robertson (Southamptom) initially treated patient 2. Mr D. DeCamp took the clinical photographs.

No benefits in any form have been received or will be received from a commercial party related directly or indirectly to the subject of this article.

\section{References}

1. Birch R. Brachial plexus injuries. J Bone Joint Surg [Br] 1996;78-B:986-92.

2. Birch R. Surgery for brachial plexus injuries. J Bone Joint Surg [Br] 1993;75-B:346-8.

3. Ochiai N, Nagano A, Sugioka H, Hara T. Nerve grafting in brachial plexus injuries: results of free grafts in 90 patients. J Bone Joint Surg [Br] 1996;78-B:754-8.

4. Htut M, Misra P, Anand P, Birch R, Carlstedt T. Pain phenomena and sensory recovery following brachial plexus avulsion injury and surgical repairs. J Hand Surg [Br] 2006;31:596-605.

5. Terzis JK, Vekris MD, Soucacos PN. Outcomes of brachial plexus reconstruction in 204 patients with a devastating paralysis. Plast Reconstr Surg 1999;104:1221-40.

6. Spinner RJ, Shin AY, Bishop AT. Update on brachial plexus surgery in adults. Techniques Hand and Upper Extremity Surgery 2005;9:220-32.

7. Birch R, Bonney G, Wynn Parry CB. Surgical disorders of the peripheral nerves. London: Churchill Livingstone, 1998:157-207.

8. Anand P, Terenghi G, Warner G, et al. The role of endogenous nerve growth factor in human diabetic neuropathy. Nat Med 1996;2:703-7.

9. Anand P, Birch R. Restoration of sensory function and lack of long-term chronic pain syndromes after brachial plexus injury in human neonates. Brain 2002;125:113-22.

10. Carlstedt T. Central nerve plexus injury. Imperial College Press 2007:78-107.

11. Hall S. The response to injury in the peripheral nervous system. J Bone Joint Surg $[\mathrm{Br}]$ 2005;87-B:1309-19.

12. Birch R, Dunkerton M, Bonney G, Jamieson AM. Experience with the free vascularized ulnar nerve graft in repair of supraclavicular lesions of the brachial plexus. Clin Orthop 1988;237:96-104

13. Gu YD, Zhang GM, Chen DS, et al. Seventh cervical nerve root transfer from the contralateral healthy side for treatment of brachial plexus root avulsion. J Hand Surg [Br] 1992;17:518-21.

14. Doi K. Management of total paralysis of the brachial plexus by the double free-muscle transfer technique. J Hand Surg Eur Vol 2008;33:240-51.

15. Landi A, Copeland SA, Parry CB, Jones SJ. The role of somatosensory evoked potentials and nerve conduction studies in the surgical management of brachial plexus injuries. J Bone Joint Surg [Br] 1980;62-B:492-6.

16. Marshall RW, De Silva RD. Computerised axial tomography in traction injuries of the brachial plexus. J Bone Joint Surg [Br] 1986;68-B:734-8.

17. Hems TE, Birch R, Carlstedt T. The role of magnetic resonance imaging in the management of traction injuries to the adult brachial plexus. J Hand Surg $[\mathrm{Br}]$ 1999;24:550-5

18. Lawson GM, Glasby MA. A comparison of immediate and delayed nerve repair using autologous freeze-thawed muscle grafts in a large animal model: the simple injury. J Hand Surg [Br] 1995;20:663-700.

19. Berman JS, Birch R, Anand P. Pain following human brachial plexus injury with spinal cord root avulsion and the effect of surgery. Pain 1998;75:199-207 